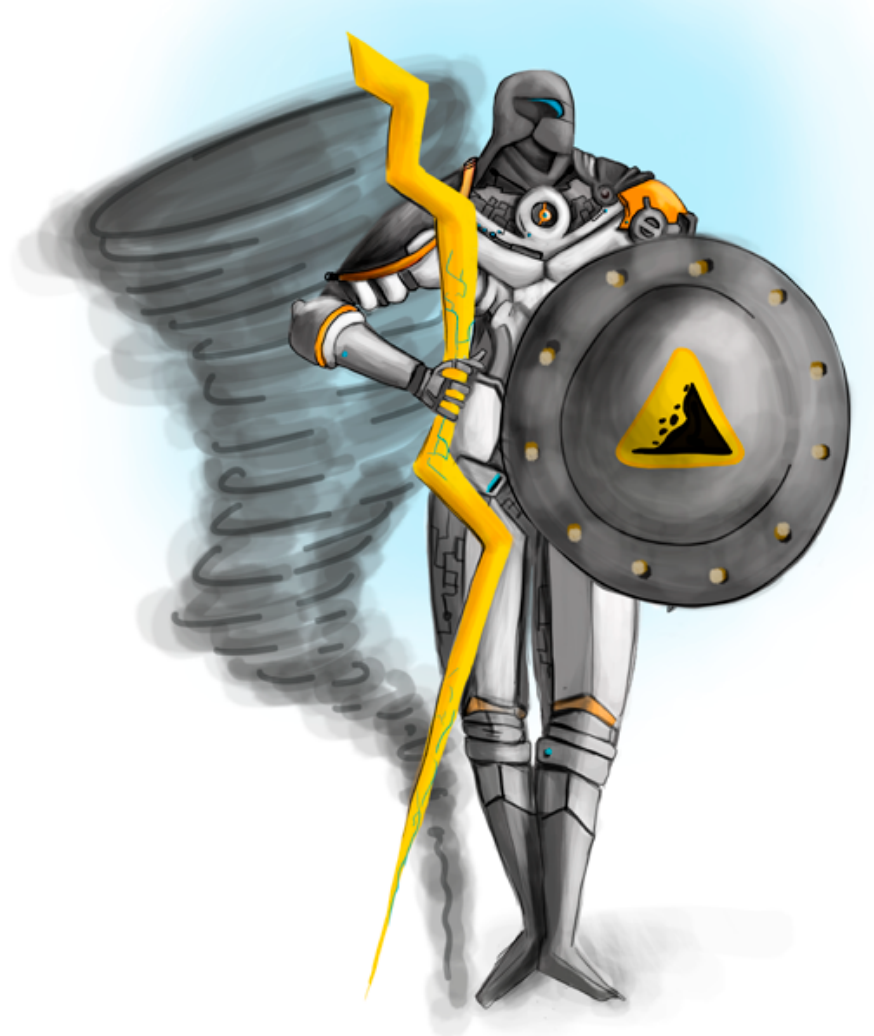

- Capítulo 4 -

\title{
Laboratorio de Riesgo Natural
}

\section{Autores}

Andrés Rodríguez Rubio. Ingeniero de sistemas, especialista en gestión de proyectos, con certificación internacional en principios de seguridad y salud en el trabajo POSH, asesor, docente y capacitador en temas de educación en seguridad y salud laboral, actualmente cursando maestría en gestión de proyectos en la universidad internacional de la Rioja. 


\section{Resumen}

Este documento registra todo el proceso de diseño, creación y construcción de un simulador de riesgos naturales para estudiantes del programa Gestión en la Salud y Seguridad en el Trabajo, del Politécnico Grancolombiano, que abarca desde el análisis de riesgo hasta la elaboración del Plan de Emergencia para la mitigación del riesgo natural. Dicho simulador sirve para trabajar la práctica o laboratorio del módulo de Higiene y salud laboral del programa profesional de Gestión en la Seguridad y Salud Laboral.

\section{Palabras claves:}

Riesgos naturales, metodología de colores para el análisis de los riesgos naturales, simulador de riesgos naturales, plan de emergencias.

\section{Introducción}

El análisis de riesgos naturales es diferente a los otros tipos de riesgos, mientras que en los otros podemos ver los efectos directos y colaterales para realizar su mitigación, en los de índole natural su gestión es más preventiva y se basa sobre hipótesis y supuestos de lo que podría suceder en caso de una posible catástrofe natural. Obviamente estas hipótesis no surgen al azar sino después de un análisis minucioso del entorno a partir de diversas metodologías como la de colores que se trabajará dentro de este proyecto.

La pregunta es cómo realizar un análisis de riesgos naturales con estudiantes mediados por tecnología, primero debe recrearse un medio cercano a la realidad que evidencie los peligros naturales para ello se crearon cinco ambientes en los cuales prevalece uno de los riesgos naturales que da la Guía Técnica Colombiana GTC. También dicho ambiente puede tener otros riesgos naturales asociados. Segundo se crearon cinco compañías de diferentes índoles (laboratorio químico, oficina, manufactura, pozo petrolero, empresa de servicios generales), las cuales se combinan de forma aleatoria para producir diversos escenarios posibles de ocurrencia para el análisis de riesgos con la metodología de colores, al final los estudiantes cuentan con veinticinco ambientes de simulación.

El proceso se diseñó de manera que el estudiante vaya en una ruta de aprendizaje, inicia con la presentación de la empresa y las condiciones 
naturales, continua con el análisis del riesgo, la clasificación y la evaluación de este, y termina, con la construcción del plan de emergencia para la compañía que es la competencia final del proceso. El plan de emergencia es el resultado del análisis de riesgos y cómo se puede mitigar anteponiéndose a la ocurrencia del desastre. De esa forma, el estudiante simula un ambiente cercano a la realidad, pero lo más importante, apropia una metodología para el análisis y mitigación del riesgo natural que es aplicable a cualquier tipo de empresa del entorno laboral.

\section{Marco teórico}

\section{Problemática que atiende}

Teniendo como premisa solventar la problemática con respecto a las prácticas de los estudiantes que existe en la modalidad virtual se planteó desde la parte académica crear ambientes virtuales de aprendizaje que permitieran replicar situaciones cotidianas sin tener necesidad de una presencia física, y teniendo claro que el objetivo primordial es acercar al estudiante a una experiencia cercana a la realidad que en determinado momento puede llegar a suceder en su quehacer diario como futuro profesional.

Para dar cuenta de esta situación de práctica estudiantil se construyó el simulador de Higiene y Seguridad Laboral, que sirve para el análisis de riesgos de la siguiente naturaleza: biológico, químico, de seguridad, físico, biomecánico y natural. Para trabajar diferentes ambientes se pensó en cinco tipos de empresas para todos los riesgos: oficina, manufactura, laboratorio químico, pozo petrolero y servicios generales.

Teniendo claras estas premisas se va a desarrollar un proceso que sirva de práctica estudiantil y de experiencia a través del cual se pretende que el estudiante identifique de manera audiovisual, con diversos materiales multimedia y ayudas digitales una herramienta que le permita identificar y analizar los riesgos naturales a fin de tomar las medidas del caso para mitigarlos.

El propósito de aprendizaje del simulador se da como resultado de un análisis de las competencias de los diferentes módulos del programa, se identifica que los estudiantes deben ser capaces de identificar, evaluar y mitigar los riesgos inherentes a un entorno laboral y a su vez los riesgos antrópicos y sociales que se puedan desencadenar a partir de la ocurrencia de un evento natural.

Para el riesgo natural en particular se inició el diseño del módulo teóricopráctico, donde la simulación diera cuenta de la parte práctica de acuerdo 
con los contenidos del módulo. Para la construcción del simulador se hizo un análisis de las metodologías existentes en la identificación de riesgos naturales y se definió optar por su practicidad y simpleza la Metodología de análisis de riesgos por colores.

Para empezar la experiencia se hizo un análisis de las herramientas disponibles en el mercado, obteniendo como resultado que no existe alguna que resulva las necesidades de formación que requiere el programa. Por tal razón, se inició el diseño desde lo pedagógico y lo conceptual para el análisis del riesgo.

En el riesgo natural se encontró la dificultad de cómo analizar los diferentes riesgos naturales (sismo, terremoto, vendaval, inundación, derrumbe y precipitaciones) definidos en la GTC. Si los riesgos dependen del ambiente en el que se encuentra la compañía crear un ambiente o escenario virtual es muy complejo porque depende de cada empresa y del ambiente natural en el que esta se ubica.

Por lo anterior se diseñaron cinco ambientes con condiciones extremas que realzan uno de los riesgos presentes en la GTC, sin embargo, puede tener uno o más riesgos asociados. Con las cinco empresas que se definieron se hace una combinación aleatoria teniendo veinticinco posibles mixturas de ambientes virtuales donde cada una tiene sus características propias haciendo que el análisis sea diferente para cada una de ellas. Lo que es común, es la aplicación de la metodología, que se vuelve el componente de apropiación para el estudiante, lo más relevante no es el tipo de empresa o el ambiente, sino la adecuada aplicación de la metodología que le permita al estudiante realizar un análisis consistente con el fin de crear una política de seguridad que se vea reflejada a través del plan de emergencias.

\section{Antecedentes}

El simulador se crea como una solución a la necesidad en las aulas, por tal razón en primera instancia se realizó la búsqueda de este en el mercado para ver que ofrecía y cómo se podía homologar en el proceso de aprendizaje de los estudiantes. La exploración no arrojó resultados satisfactorios. Los simuladores encontrados son muy básicos y de nivel introductorio puesto que no llegan al punto del análisis del riesgo y menos a la formulación de controles o planes adecuados para la mitigación.

Dentro de la indagación se encontró un juego que sirve como emulador de riesgos naturales y recrea los siguientes escenarios: terremoto, inundación, huracán, tsunami e incendio forestal. Se da un presupuesto inicial al usuario el 
cual tiene como objetivo o meta salvar vidas tratando de construir viviendas reforzadas, barreras, albergues y hoteles previendo un desastre inevitable. Al final el jugador obtiene puntos según la cantidad de vidas que salve. El juego tiene niveles, de acuerdo con este nivel varía el presupuesto asignado y la población total. La gran limitante es que no se hace un análisis del riesgo, ni propone un plan de emergencia, solo se limita a construir barreras naturales o viviendas a fin de alojar a la mayor cantidad de población posible. Se puede consultar el juego en: http://www.stopdisastersgame.org/es/playgame.html
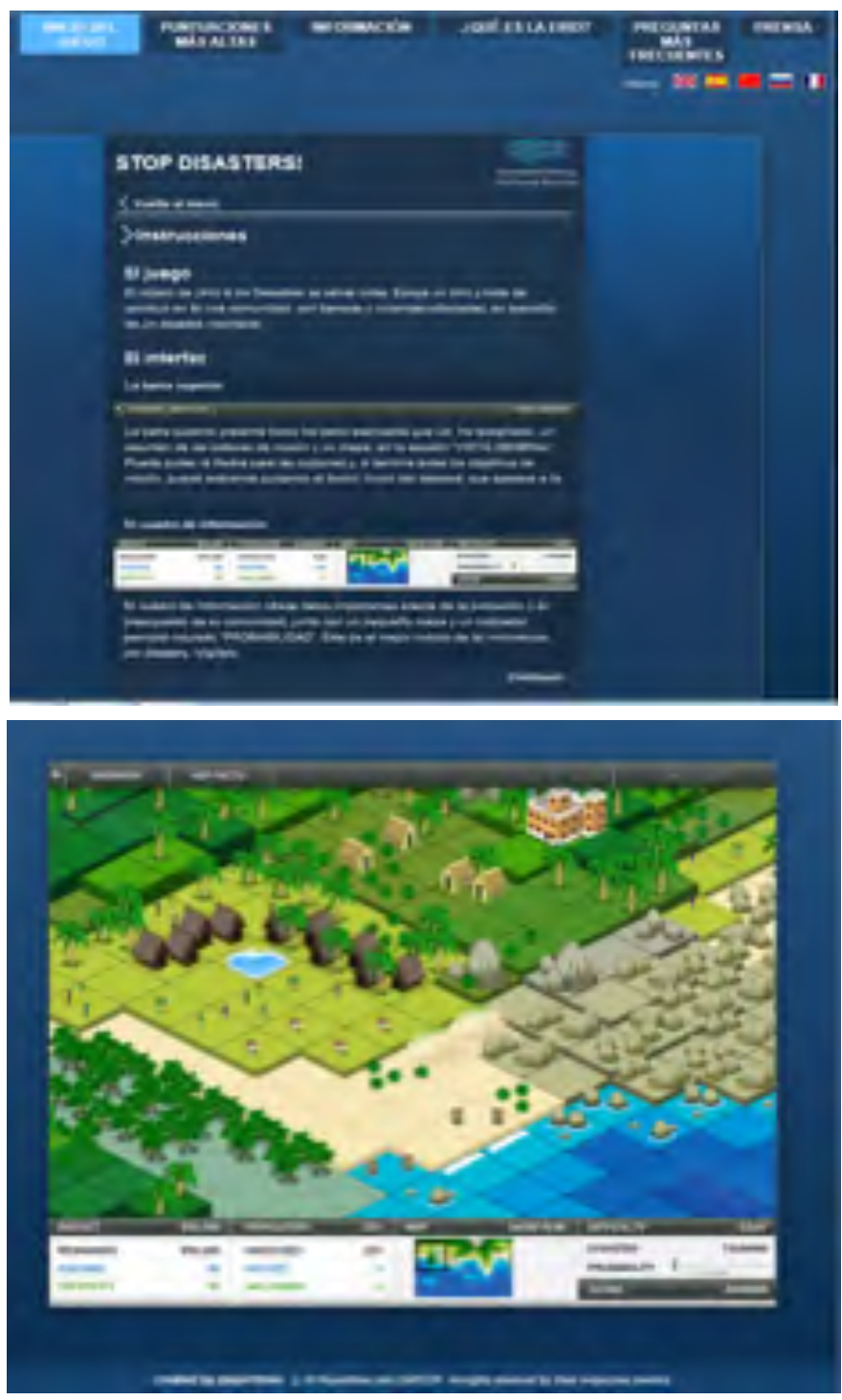

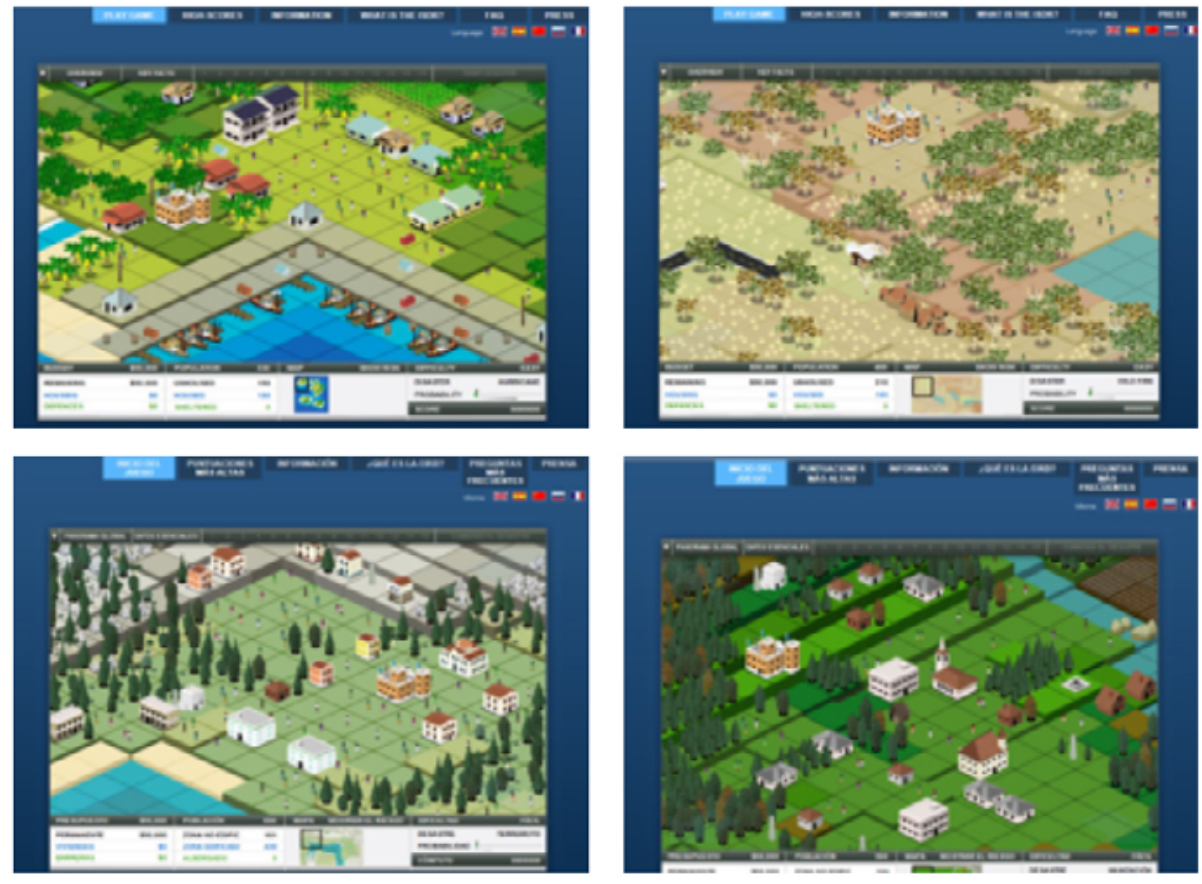

Figura 1. Interfaces Stop Disasters. Interfaces de: Inicio, Tsunami, Huracán, Incendio Forestal, Terremoto, Inundación.

Fuente: Pantallazos Stop Disasters, en: http://www.stopdisastersgame.org/en/home.html

Por otro lado, se identifcó una empresa en Estados Unidos que realiza capacitaciones a través de simuladores, son ajustables a cada situación no solo para la atención de desastres naturales sino también para la capacitación de cuerpo de bomberos y de policía, uno de sus focos es entrenar el personal de las empresas para afrontar situaciones de desastres tales como inundaciones, terremotos e incendios forestales; adicional a ello, se incorporan algunas actividades de respuesta como búsqueda, rescate de edificios derrumbados y áreas inundadas, manejo de víctimas en masa, provisión de refugio y evacuación a gran escala.

La empresa se llama ETC simulation y el simulador se denomina Advanced Disaster Management Simulator (ADMS), su centro de operaciones se encuentra en Orlando, FL, y trabajan con diferentes institutos de simulación y formación. ADMS fue desarrollado después de un desastre en Manchester, Inglaterra, cuando la Autoridad de Aviación Civil del Reino Unido encargó al Cranfield Institute of Technology para estudiar las necesidades de entrenamiento de 
rescate de incendios en aeropuertos. El estudio resultante encontró que se requería un sistema de entrenamiento para el comando y control de incidentes en un desastre (ETC simulation, 2017)

El sistema de capacitación de simulación se está utilizando con éxito en el desarrollo, capacitación y evaluación de planes de respuesta de las organizaciones de gestión de emergencias en todo el mundo. Consultar en: http://www.trainingfordisastermanagement.com/applications/disasterpreparedness-and-response/

Una de las desventajas que tiene el simulador es que funciona únicamente en modalidad presencial en la sede de la compañía, adicional a ello, los costos por cada usuario son de aproximadamente USD 4000. Es una aplicación de realidad virtual que funciona en cabinas donde el usuario interactúa con gafas, con guantes de realidad virtual y la idea es que dé respuesta a lo que puede suceder durante el desastre. Es un entrenamiento recomendable para una brigada y se aleja un poco del objeto de estudio que pretende el simulador de este proyecto, el cual se centra en la prevención y preparación de una empresa para un posible desastre de acuerdo con el riesgo natural más eminente que se ve expuesta según el ambiente natural en el que se encuentra.

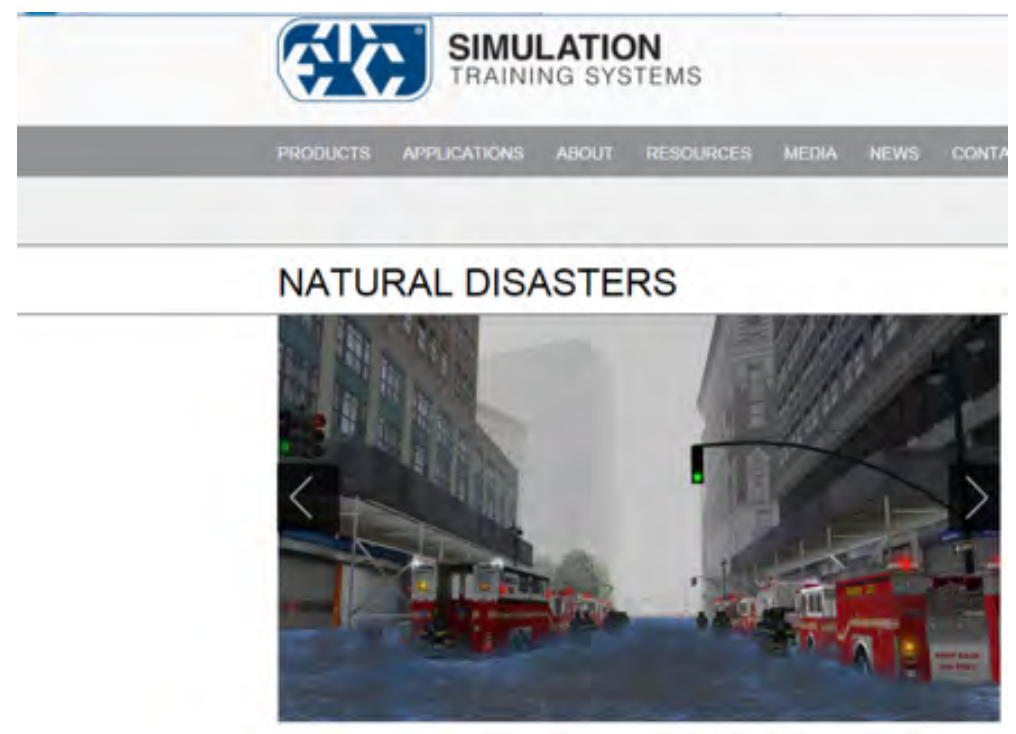

Figura 2. Interfaz ETC Simulation Training Systems.

Fuente: Pantallazos ETC Simulation Training Systems en: http://www.

trainingfordisastermanagement.com/applications/disaster-preparedness-and-response/ 
En la indagación no se encontraron más herramientas similares, por lo tanto, el simulador que se presenta en esta experiencia es único en su especie.

\section{Estrategia de aprendizaje}

Según el Fondo para Prevención y Atención de Emergencias - FOPAE (2012): Se define el análisis o evaluación de riesgos como el proceso de estimar la probabilidad de que ocurra un evento no deseado con una determinada severidad o consecuencias en la seguridad, salud, medio ambiente $y / 0$ bienestar público. A partir del 2014 el FOPAE cambió al Instituto Distrital de Gestión de Riesgos - IDIGER quien retomando lo del FOPAE normativiza que todas las organizaciones deben elaborar un Plan de Emergencia y Contingencia que permita prevenir y mitigar riesgos, atender los eventos con la suficiente eficacia, minimizando los daños a la comunidad y al ambiente y recuperarse en el menor tiempo posible (FOPAE, 2012).

Asimismo, el decreto el Decreto 0472 de 2015 el cual reglamenta los criterios de graduación de las multas por infracción a las normas de Seguridad y Salud en el Trabajo y Riesgos Laborales, se señalan normas para la aplicación de la orden de clausura del lugar de trabajo o cierre definitivo de la empresa y paralización o prohibición inmediata de trabajos o tareas y se dictan otras disposiciones. (Ministerio de Trabajo, 2015)

De acuerdo con esta determinación se vuelve una necesidad para el programa de Profesional de la Gestión de Seguridad y Salud Laboral del Politécnico Grancolombiano incorporar en su plan de estudios el análisis de riesgos naturales dentro de sus módulos de Higiene y Seguridad Laboral. Por esta razón, se pensó en construir un espacio de práctica que evidencie la adquisición de las competencias por parte de los estudiantes en el análisis de diferentes tipos de riesgos, entre ellos los naturales. Por tal motivo, todos los simuladores del programa se trabajan de manera teórico-práctica en los diferentes módulos.

El escenario de práctica se estructuró para cinco organizaciones diferentes con el fin de cubrir diversos campos laborales donde el futuro profesional puede desenvolverse, estos espacios son: oficina, manufactura, laboratorio químico, pozo petrolero y, prestadora de servicios de aseo y generales. El pensamiento se hizo de forma modular siguiendo la metáfora LEGO, que aplica para los objetos de aprendizaje. Según Hodgins (2000), un objeto de aprendizaje es: “... una colección de objetos de información ensamblada usando metadatos para corresponder a las necesidades y personalidad de un aprendiz en particular. 
Múltiples objetos de aprendizaje pueden ser agrupados en conjuntos más grandes y anidados entre sí para formar una infinita variedad y tamaños".

Para esta experiencia educativa se aplica la metáfora LEGO, extrapolada a un simulador donde cada empresa funciona de forma modular, para que en posteriores desarrollos pueda implementarse si se requiere otras empresas. De manera similar, para el simulador de riesgo natural se proponen cinco ambientes naturales cada uno asociado a un riesgo de índole natural (vendaval, derrumbe, inundación, sismo y terremoto, precipitación), de tal manera que con cinco empresas y cinco ambientes combinándolos de forma aleatoria podemos generar veinticinco escenarios de análisis diversos que si se agregan más empresas o más ambientes se pueden multiplicar las posibilidades para los estudiantes.

Para ampliar las posibilidades del análisis y evaluación de riesgos, cada empresa tiene unas características diferentes en las cuales se manifiestan diversos tipos de amenazas, de igual manera, cada una provee un análisis de riesgos diferente. Tal diferencia tiene un objetivo pedagógico importante y es la apropiación de una metodología para el análisis, pues si el estudiante trabaja un método de análisis adecuado lo podrá aplicar en diferentes circunstancias, algo que no podría realizarse si el simulador se centrara en una empresa y no en una metodología.

El proceso se estructuró para que el simulador le asigne al estudiante, al azar, una empresa en un ambiente determinado. El reto educativo es que elabore para dicha empresa. de acuerdo con las condiciones naturales del entorno, el Plan de Emergencias que contiene el análisis de riesgos con la metodología de colores que se tratará más adelante, el PON, la ubicación de extintores, las rutas de evacuación y puntos de encuentro, la creación de brigadas, las capacitaciones generales al personal y las específicas para las brigadas, las recomendaciones adicionales para un caso de desastre.

El estudiante debe hacer un análisis de amenazas, detectar los riesgos naturales, los riesgos antrópicos y sociales, con el fin de que sea lo más próximo a un escenario real. De igual manera, debe cumplir todos los pasos para el análisis, desde la identificación de las amenazas, la evaluación y posterior construcción del plan de emergencias, que en últimas es trabajo final que entrega al tutor, quien juega el rol de la empresa que recibe el plan de emergencias y retroalimenta de acuerdo con las orientaciones que da el evaluador de riesgos (estudiante).

Una parte fundamental de la estrategia pedagógica es que el estudiante 
argumente sus decisiones, ya que en el análisis de riesgos no hay una solución única, por el contrario, depende más del análisis asertivo y la correcta toma de decisiones por parte del evaluador o estudiante.

El propósito final es que el estudiante pueda prevenir o mitigar un riesgo natural en caso de una eventual presencia de un riesgo o desastre. Según Larraín y Simpson (1994) la mitigación son los "procedimientos adoptados por la población con el objeto de minimizar los efectos de un evento natural extremo". Wilches-Chaux (en Maskrey, 1993) define la prevención como "la reducción del peligro o la amenaza". Es complejo reducir a cero un peligro natural ya que no existe una tecnología en la actualidad para prevenir o controlar un terremoto, un huracán, o cualquier evento natural. (Rojas O. y Martínez C., 2011). Entonces lo único que se puede hacer es crear procesos de prevención y manejo del desastre en caso de ocurrencia.

\section{Modelo para la simulación}

Existen varias metodologías que permiten realizar el análisis de los riesgos naturales, cada una de ellas tiene sus ventajas, la elección de la más apropiada depende de la cantidad de información disponible y del nivel de detalle que se desee alcanzar. Por lo tanto, se van a exponer las tres más utilizadas y después se presentarán los argumentos de la metodología que se seleccionó de acuerdo con las necesidades de la simulación.

Metodología de Análisis preliminar de riesgos basados en el Programa de Concientización y Preparación para Emergencias a Nivel Local (APELL) el cual fue dado a conocer en 1988 por el Centro de Actividades del Programa de Industria y Medio Ambiente (UNEP IE/PAC) del Programa de las Naciones Unidas, en cooperación con los gobiernos y la industria química. Esta metodología no pretende realizar un análisis de riesgo exhaustivo de la Organización, pero sí obtener un análisis primario que permita conocer de manera general y anticipada los principales riesgos de una instalación industrial. El énfasis se realiza en los accidentes industriales que representen una amenaza potencial para las personas, la propiedad y el ambiente, para que a través de este conocimiento las autoridades tengan mayores elementos de juicio para establecer y mejorar la preparación para situaciones de emergencia. (PNUMA, 2003).

Después de revisar esta metodología minuciosamente se concluyó que no es la ideal para ser aplicada a las diferentes empresas que se tienen para el 
simulador, porque se centra en las empresas químicas o generadoras de impactos ambientales. Para el proyecto se requería una metodología más genérica con un rango amplio de aplicación.

Método Semicuantivativo también denominado Gross Hazard Analysis (GHA) está orientado a establecer una visión total de la posición y peso relativo de los riesgos dentro de un sistema particular. Esta metodología del tipo semicuantitativa, permite establecer criterios homogéneos para la toma de decisiones. Se basa en criterios y procedimientos requeridos para realizar de una manera costo-beneficio la gestión de los riesgos relacionados con la realización de cualquier actividad, con el fin de salvaguardar la integridad de las personas, las instalaciones, las propiedades, el medio ambiente, la imagen de la empresa, institución o actividad económica que se desarrolle. (FOPAE, 2012)

El fin de esta metodología es tratar de encontrar los impactos que podrían tener al momento de la materialización de un riesgo basado en una escala de frecuencia de seis posibilidades con las que se puede medir la gravedad de las consecuencias en cuanto a impacto humanos, impacto ambiental, impacto operacional, impacto económico e impacto institucional. También se centra en conocer las posibles repercusiones que pueden darse a partir de la materialización de un riesgo al interior de la empresa y como podría este afectar no solo a la empresa sino a su entorno. Se centra en cómo un riesgo interno en la compañía puede afectar el entorno, y no cómo el entorno puede volverse un riesgo para las personas de la compañía, por lo tanto, no se aplica de manera apropiada a los riesgos de tipo natural.

Metodología de análisis de riesgos por colores permite desarrollar análisis de amenazas y análisis de vulnerabilidad de personas, recursos, sistemas y procesos, con el fin de determinar el nivel de riesgo a través de la combinación de los elementos anteriores, con códigos de colores. Asimismo, permite formular las acciones de prevención, mitigación y respuesta que contemplan los planes de emergencia. (FOPAE, 2012)

La metodología por colores fue la que se adoptó para este proyecto porque puede utilizarse en organizaciones, empresas, industrias e instalaciones de todo tipo. Además, va acorde con el método de análisis de un riesgo que inicia estableciendo las amenazas, identifica su fuente $u$ origen, la posible magnitud de las consecuencias, estudia el impacto y a partir de ello toma las medidas pertinentes a fin de mitigar el riesgo. Otro criterio de selección se debe a que es la metodología recomendada por Fondo de Prevención y Atención de Emergencias (FOPAE). 


\section{¿Cómo funciona la metodología por colores?}

Lo primero que se debe hacer es realizar un análisis de amenazas que dependerá del tipo de empresa, su ubicación geográfica, de sus instalaciones físicas o de su entorno; dichas amenazas se pueden clasificar en naturales, antrópicas no intencionales o sociales, dependiendo de origen de ocurrencia. Una amenaza antrópica es la consecuencia de una natural, se refiere a un posible riesgo que puede generarse con la presencia de un desastre natural regularmente son ocasionados por el ser humano o por desgaste de la naturaleza como deforestación o erosión, y puede ser un incendio, un deslizamiento de tierra, etc. En cambio, los riesgos sociales se refieren a comportamientos de los individuos ante la ocurrencia de un desastre.

Tabla 1. Identificación de amenazas.

\begin{tabular}{|l|l|l|}
\hline NATURALES & ANTRÓPICAS NO INTENCIONALES & SOCIALES \\
\hline $\begin{array}{l}\text { Se deben listar las } \\
\text { amenazas naturales } \\
\text { encontradas }\end{array}$ & $\begin{array}{l}\text { Se deben listar las amenazas } \\
\text { antrópicas no intencionales } \\
\text { encontradas }\end{array}$ & $\begin{array}{l}\text { Se deben listar las amenazas } \\
\text { sociales encontradas }\end{array}$ \\
\hline
\end{tabular}

Fuente: Creación propia.

Como parte de la identificación se debe realizar la descripción, definir si es interna o externa a la empresa y, por último, calificar la amenaza. Para ello, se puede utilizar un cuadro como el que se describe a continuación y que se replica dentro del simulador. Ver tabla 2.

Tabla 2. Calificación de las amenazas.

\begin{tabular}{|c|c|c|c|c|c|}
\hline AMENAZA & INTERNO & EXTERNO & $\begin{array}{l}\text { DESCRIPCIÓNDE } \\
\text { LA AMENAZA }\end{array}$ & CALIFICACIÓN & COLOR \\
\hline \multirow{3}{*}{$\begin{array}{l}\text { Se deben } \\
\text { colocar una } \\
\text { a una las } \\
\text { posibles } \\
\text { amenazas } \\
\text { tanto } \\
\text { naturales } \\
\text { como } \\
\text { antrópicasy } \\
\text { sociales. }\end{array}$} & \multirow{3}{*}{$\begin{array}{l}\text { Se debe } \\
\text { especificar } \\
\text { sila } \\
\text { amenaza } \\
\text { es de } \\
\text { origen } \\
\text { interno. }\end{array}$} & \multirow{3}{*}{$\begin{array}{l}\text { Se debe } \\
\text { especificar } \\
\text { sila } \\
\text { amenaza } \\
\text { es de } \\
\text { origen } \\
\text { externo. }\end{array}$} & \multirow{3}{*}{$\begin{array}{l}\text { Esta debe ser lo } \\
\text { más detallada } \\
\text { posible, } \\
\text { incluyendo } \\
\text { datos relevantes, } \\
\text { la fuente o } \\
\text { estudios que } \\
\text { sustente la } \\
\text { posibilidad o no } \\
\text { de ocurrencia. }\end{array}$} & $\begin{array}{l}\text { POSIBLE: Nunca } \\
\text { ha sucedido. }\end{array}$ & Verde \\
\hline & & & & $\begin{array}{l}\text { PROBABLE: Ya } \\
\text { ha ocurrido. }\end{array}$ & Amarillo \\
\hline & & & & $\begin{array}{l}\text { INMINENTE: } \\
\text { evidente, } \\
\text { detectable. }\end{array}$ & Rojo \\
\hline
\end{tabular}

Fuente: Creación propia. 
Luego de encontrar, describir y calificar las amenazas, se procede a realizar el análisis de vulnerabilidades que contempla los tres elementos de posible exposición: personas, recursos, sistemas y procesos (se toma como uno solo), cada uno de ellos debe tener un formato individual que a través de preguntas buscan evaluar de manera cualitativa. Sin embargo, dicha evaluación también se hace de forma cuantitativa asignándole un valor con el fin de que el evaluador determine si es o no vulnerable frente a la amenaza descrita. Ver tabla 3.

Tabla 3. Calificación de las vulnerabilidades.

\begin{tabular}{|c|c|c|c|c|c|}
\hline \multirow{2}{*}{$\begin{array}{l}\text { PUNTOA } \\
\text { EVALUAR }\end{array}$} & \multicolumn{3}{|l|}{ RESPUESTA } & \multirow[t]{2}{*}{ CALIFICACIÓN } & \multirow[t]{2}{*}{ OBSERVACIONES } \\
\hline & PUNTOAEVALUAR & PUNTOAEVALUAR & PUNTO AEVALUAR & & \\
\hline $\begin{array}{l}\text { Se realiza un } \\
\text { conjunto de } \\
\text { preguntas que } \\
\text { se formulan } \\
\text { en la primera } \\
\text { columna, las } \\
\text { cuales orientan la } \\
\text { calificación final. }\end{array}$ & $\begin{array}{l}\text { Se marca con X } \\
\text { si la respuesta } \\
\text { es "SI". }\end{array}$ & $\begin{array}{l}\text { Se marca con X } \\
\text { si la respuesta } \\
\text { es "NO". }\end{array}$ & $\begin{array}{l}\text { Se marca con } X \\
\text { si la respuesta es } \\
\text { "PARCIAL". }\end{array}$ & $\begin{array}{l}\text { Si es "SI" = } 1 \\
\text { Si es "NO" =0 } \\
\text { Si es "PARCIAL" } \\
=0,5\end{array}$ & $\begin{array}{l}\text { Estas permiten } \\
\text { identificar } \\
\text { aspectos de } \\
\text { mejora que van a } \\
\text { ser contemplados } \\
\text { en los planes de } \\
\text { acción. }\end{array}$ \\
\hline \multicolumn{4}{|c|}{ Promedio Vulnerabilidad } & & \\
\hline
\end{tabular}

Fuente: Creación propia.

La interpretación del promedio se da de acuerdo con un color, el color rojo corresponde a alta, es decir hay una vulnerabilidad inminente. Amarillo significa media, existe la vulnerabilidad, pero se tienen medidas para mitigarla. Verde es baja, significa que en ese momento no representa riesgo. Ver la tabla 4.

Tabla 4. Interpretación de las vulnerabilidades por cada elemento.

\begin{tabular}{|l|l|l|}
\hline \multicolumn{1}{|c|}{ RANGO } & \multicolumn{1}{|c|}{ INTERPRETACIÓN } & \multicolumn{1}{c|}{ COLOR } \\
\hline Siel promedio se encuentra entre 0 y 0,33 & ALTA & ROJO \\
\hline Si el promedio se encuentra entre 0,34 y 0,67 & MEDIA & AMARILLO \\
\hline Si el promedio se encuentra entre 0,68 y 1 & BAJA & VERDE \\
\hline
\end{tabular}

Fuente: FOPAE (2012). 
En el simulador dichas preguntas se realizan a un avatar que tiene el papel de ser el gerente de la empresa, este va a ser el mismo en todas las empresas, sin embargo, sus respuestas son distintas para que cada análisis sea diferente entre sí.

El paso siguiente es crear el diamante del riesgo, el cual resume la combinación de amenazas y vulnerabilidades de cada uno de los tres elementos expuestos analizados. Se toma la amenaza y se analiza en cada uno de los factores de exposición. Ver figura 3.

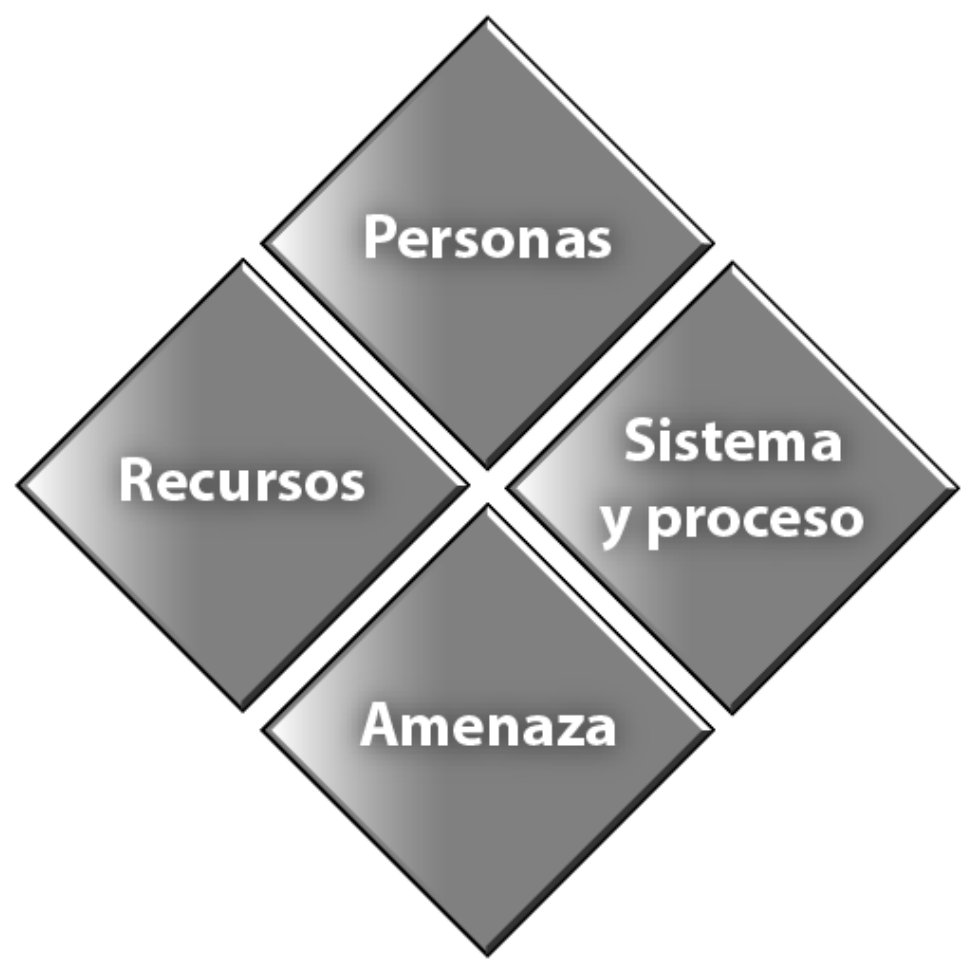

Fuente: Creación propia.

Cada uno de los rombos tiene un color y fue asignado de acuerdo con el análisis desarrollado. Con base en el diamante se debe calificar el riesgo. Ver la figura 4. 


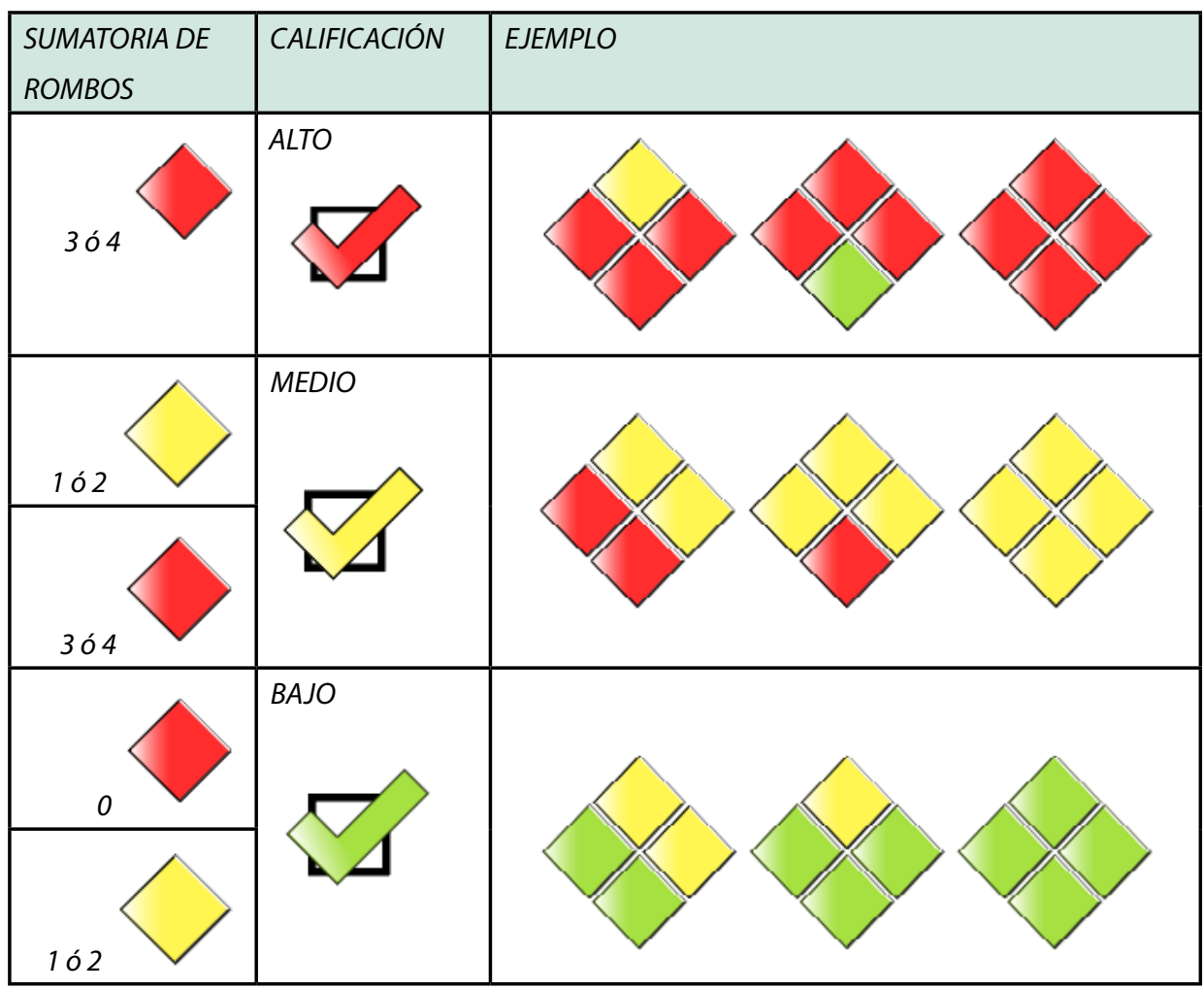

Figura 4. Calificación nivel de riesgo.

Fuente: FOPAE (2012)

Si los rombos rojos son iguales o superiores a tres se determina que el riesgo es alto y deben ejecutarse acciones inmediatas a fin de intentar mitigarlo. Si los rombos rojos son inferiores o iguales a dos o los rombos amarillos son iguales a tres o superiores se determina que el riesgo es medio, lo que significa que se deben tomar medidas a corto o mediano plazo a fin de que este no se vuelva alto; de todos modos, si existe la presencia de un rombo rojo se considera un riesgo medio. Si no hay rombos rojos significa que el riesgo es bajo, si hay presencia de rombos amarillos quiere decir que se deben realizar acciones preventivas a mediano y largo plazo.

Con la información plasmada en el rombo, se procede a diligenciar el consolidado de análisis del riesgo a fin de realizar un análisis horizontal de cada uno de ellos y demostrar a la empresa de manera gráfica y resumida las vulnerabilidades que tiene cada elemento analizado para que prioricen la intervención de las amenazas que se encontraron dentro del proceso. Ver tabla 5. 
Tabla 5. Consolidado de análisis del riesgo.

\begin{tabular}{|c|c|c|c|c|c|c|c|c|c|c|}
\hline \multirow{2}{*}{ TIPO } & \multirow{2}{*}{ ơ } & \multirow{2}{*}{ 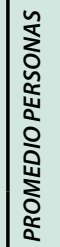 } & \multirow{2}{*}{ 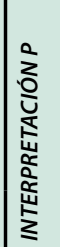 } & \multirow{2}{*}{ 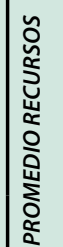 } & \multirow{2}{*}{ 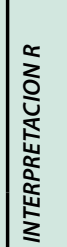 } & \multirow{2}{*}{ 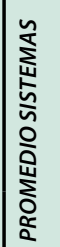 } & \multirow{2}{*}{ 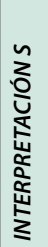 } & \multirow{2}{*}{$\begin{array}{c}\text { ANTECEDENTES } \\
\text { - CAUSASO } \\
\text { FUENTESDE } \\
\text { RIESGO }\end{array}$} & \multicolumn{2}{|c|}{ NIVEL DE RIESGO } \\
\hline & & & & & & & & & DIAMANTE & INTERPRETACIÓN \\
\hline NATURALES & & & & & & & & & & \\
\hline $\begin{array}{l}\text { ANTRÓPICOS NC } \\
\text { INTENCIONALES }\end{array}$ & & & & & & & & & & \\
\hline SOCIALES & & & & & & & & & & \\
\hline
\end{tabular}

Fuente: FOPAE (2012)

El simulador diligencia esta tabla con la información que va digitando el usuario en cada uno de los escenarios. Adicionalmente, a partir de la información obtenida se pude realizar la priorización de las amenazas colocando en la parte superior las de mayor nivel de riesgo con el fin de definir las medidas de prevención o mitigación según sea el caso. La priorización de amenazas es el paso final de la Metodología de análisis de riesgo por colores. Ver tabla 6.

Tabla 6. Priorización de amenazas y medidas de intervención.

\begin{tabular}{|l|l|l|l|}
\hline AMENAZA & MEDIDA DE INTERVENCIÖN & \multicolumn{2}{|c|}{ TIPO DE MEDIDA } \\
\cline { 3 - 4 } & & PREVENCIÓN & MITIGACIÓN \\
\hline & & & \\
\hline
\end{tabular}

Fuente: FOPAE (2012)

La priorización es una parte fundamental en la creación del Plan de Emergencias, cuyo objetivo es definir los procedimientos, procesos y responsables a fin de poder responder de manera oportuna y coordinada en caso de la ocurrencia de una emergencia; es le permite al simulador recrear una situación lo más parecida a la realidad, para ello se incluyó dentro del análisis riesgos antrópicos como incendio, explosión y fallas en equipos. También accidentes personales y comportamientos no adaptativos que corresponden a riesgos sociales, esto con el fin de realizar un análisis integral y tratar de emular la realidad ante la ocurrencia de un desastre natural. 
A partir del análisis del ambiente, como se hace en un entorno real, se llega a la construcción del Plan de Emergencias, por eso se incluyó dentro del proceso de simulación la creación del PON (Planes Operativos Normalizados), ubicación de extintores, creación de rutas de evacuación, ubicación de puntos de encuentro, creación de brigadas de evacuación, primeros auxilios, comunicación y prevención de incendios. También se deben sugerir capacitaciones al personal y brindar recomendaciones generales y por cada riesgo encontrado.

De acuerdo con los resultados de la evaluación por la Metodología de colores se procede a la elaboración del Plan de Emergencias que contiene las estrategias de acción y mitigación, dicho plan se inicia con la construcción del PON, este se hace para cada amenaza sin importar su calificación dentro de la metodología, para cada uno de ellos se toma el antes, durante y después a través de un gráfico. Para ello, el simulador tiene un espacio de diagramación apropiado para que el estudiante elabore dicho gráfico. En un escenario posterior el estudiante puede definir los responsables, esto es posterior a la creación de las brigadas.

El siguiente paso en la elaboración del Plan de Emergencias es la ubicación de extintores. El simulador le muestra al estudiante el plano de la empresa que le correspondió y como usuario tiene la posibilidad de ubicarlos arrastrando el extintor sobre el plano; además, le ofrece la posibilidad según la empresa de utilizar diferentes tipos de extintores, después debe justificar sus decisiones.

Para continuar el proceso el estudiante debe, a partir ya no solo con el plano interno sino con la vista superior de las instalaciones, interior y exterior; dibujar las rutas de evacuación y los puntos de encuentro para la empresa y argumentar sus decisiones.

El simulador le ofrece al estudiante un grupo de personas que laboran en la empresa, dichas personas tienen una ficha de presentación y a partir de la misma debe conformar las brigadas teniendo en cuenta el perfil de cada uno, el cargo, el tipo de contrato y la antigüedad en la compañía. Es muy importante que sus decisiones sean tomadas con criterio y sean argumentadas.

Con las brigadas creadas, debe proponer los diferentes tipos de capacitación para el personal y para las brigadas si lo requiere. Para finalizar el proceso debe realizar sugerencias o recomendaciones a la empresa con el fin de mitigar los posibles riesgos o las posibles consecuencias en caso de un desastre. 


\section{Desarrollo de la experiencia de aprendizaje}

Luego de entender la necesidad que existía en el entorno virtual, y procurando tener un acercamiento a la realidad de lo que puede suceder a nivel empresarial en la ocurrencia de un desastre natural, se identificaron cuáles son los aspectos más relevantes a tener en cuenta en cuanto a ubicación geográfica, tipo de empresas que de acuerdo a su tipología podían tener un manejo diferente y las condiciones generales de las zonas aledañas, adicional a ello realizar la inclusión de los planos de la empresa a fin de identificar los puntos clave dentro del proceso de creación de un plan de emergencias tales como la ubicación de extintores, la creación de rutas de evacuaciones y los puntos de encuentro.

Teniendo claras estas premisas y sabiendo que el objetivo es planear el proceso de simulación, se inició con la identificación de los escenarios que cumplieran con las características más relevantes en cuanto a la ocurrencia de un evento natural; tratando siempre de hacer evidente el riesgo al que se estaría expuesto en esta ubicación sin omitir que cualquiera de las empresas que se debían crear podría ubicarse en este espacio a fin de realizar el proceso de elaboración del plan de emergencias.

Luego de tener claras las condiciones de ubicación, se crearon cinco empresas que pudieran acoplarse sin problema a los espacios creados. Dichas empresas debían tener también condiciones especiales con el fin tratar de hacer que siempre estuviera inmerso el riesgo natural y que este desencadenara otros riesgos para hacer que la experiencia sea lo más cercana a la realidad. Adicional a ello, se hizo una descripción general del tipo de empresa con su objeto social, así como, información acerca de la cantidad de empleados.

Teniendo claras las consideraciones de ubicación y tipo de empresa, se crearon los planos internos de cada una de ellas pensando siempre en tener condiciones que favorecieran la identificación de las zonas más vulnerables y de los espacios que deberían destinarse para la ubicación de extintores o elementos de seguridad, así como también previendo que pudiesen hacerse notorias las zonas que pudieran destinarse como puntos de encuentro.

Dentro del proceso de creación, también se realizó una descripción de los personajes que estarán presentes a fin de tener en cuenta las características fisiológicas y psicológicas que permitirán tener una idea de algunas de 
las personas que hacer parte de la empresa y que podrían jugar un papel indispensable en la creación de las brigadas de emergencias, cada uno de ellos cumple un rol específico dependiendo la empresa en la que se esté realizando la simulación.

Finalmente, se inicia con la creación de los guiones en los que se tratarán de plasmar las situaciones más relevantes a las que se verá enfrentado el estudiante en la identificación de los riesgos naturales y los desencadenantes de estos a lo largo de su carrera profesional, también serán el componente clave para poder realizar un óptimo proceso de identificación y creación del plan de emergencias.

Por último, se construye la guía metodológica para la simulación y los tutoriales de manejo que van incluidos con el personaje de ayuda denominado Bernard que es transversal a todos los simuladores de riesgos laborales que se producen en la Institución Universitaria Politécnico Grancolombiano.

\section{Resultados obtenidos}

El resultado de esta primera versión de la herramienta son cinco ambientes naturales cada uno asociado a un riesgo, aumentando la probabilidad de ocurrencia sin descartar el análisis en los demás riesgos naturales. En estos cinco ambientes de manera aleatoria se ubica una de las cinco empresas y se generan diversos casos con diversas posibilidades de análisis. La herramienta a su vez le permite al estudiante simular y obtener un documento final para entregar al tutor, generando así un espacio de práctica pues la metodología apunta a que el estudiante sea capaz de elaborar para una empresa un Plan de emergencias, en este caso lo aplica según el escenario de simulación, pero la construcción de todo el proceso es el propósito central de aprendizaje, es decir, el estudiante puede aplicar la metodología en un escenario real en cualquier tipo de organización.

\section{Conclusiones}

A lo largo de la creación de este espacio de aprendizaje se mantuvo como objetivo desarrollar una herramienta que de manera práctica ayude y oriente 
a los estudiantes en la identificación de vulnerabilidades y riesgos ante un desastre natural, y con base en ello, crear y proponer planes de acción a fin de poder tener el menor impacto posible.

Con los datos entregados y generados en el simulador, se puede realizar un análisis claro y conciso que tiene como único fin dar una guía ante la creación de un plan de emergencia de riesgos naturales a nivel empresarial; esto es objetivo primordial del simulador pues pretende ser una herramienta que permite de manera intuitiva aplicar la metodología de colores con el fin de realizar un análisis a cualquier empresa en cualquier escenario y ante las condiciones a las que puede verse enfrentada una empresa en el mundo real.

Así pues, se logró tener en una sola herramienta todas las variables que se deben tener dimensionadas ante la creación de un plan de emergencias y poder brindar las recomendaciones necesarias o entregar crear planes de mitigación que sirvan para minimizar el riesgo.

\section{Bibliografía o referencias}

Colombia, Ministerio del Trabajo (17 de marzo de 2015) Decreto 0472 de 2015. Recuperado de: http://www.leyex.info/leyes/Decreto472de2015.pdf

ETC simulation (2017). Historia de la compañía. Recuperado de: http://www. trainingfordisastermanagement.com/about/etc-simulation/

Fondo De Prevención y Atención De Emergencias - FOPAE (2012). Metodologías de análisis de riesgo, Guía para elaborar planes de emergencia y contingencias. Bogotá, FOPAE. Recuperado de: http://www.ridsso.com/documentos/muro/ fe6dd4f800e4ed2467827680f51e2ae8.pdf

Hodgins, W. (2000). Into the future. A vision paper. 2000 [en línea]. Recuperado de: http:// www.learnativity.com/download/MP7.PDF

Larraín P. y Simpson P. (1994) Percepción y prevención de catástrofes naturales en Chile. Universidad Católica de Chile, Santiago.

Programa de las Naciones Unidas para el Medio Ambiente - PNUMA y Universidad de Guadalajara (2003). Identificación y evaluación de riesgos en una comunidad local. México D.F., Universidad de Guadalajara. Recuperado de: https://issuu.com/julianrivas/ docs/namee335a4 
Rojas O. y Martínez C. (2011) Riesgos naturales: evolución y modelos conceptuales. Revista Universitaria de Geografía [en línea]. Disponible en: $<$ http://www.redalyc.org/articulo. oa?id=383239103004> ISSN 0326-8373.

Wilches-Chaux, G. (1993) La vulnerabilidad global. En: Maskrey, A (Comp.). Los Desastres No Son Naturales. La Red.

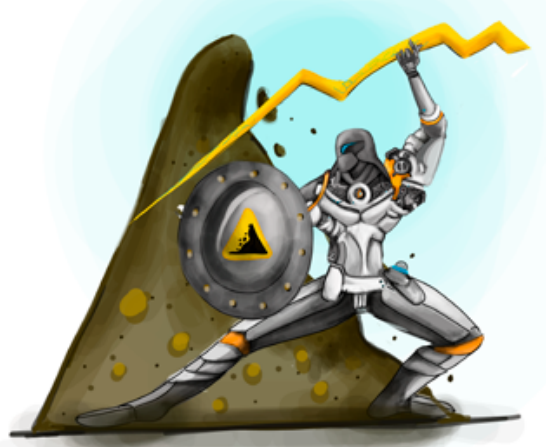

